\title{
Magnetic field enhanced nano-tip fabrication for four-probe STM studies
}

\author{
J E McKendry ${ }^{1}$, C S Allen ${ }^{1}$, K Critchley ${ }^{2}$, M Ł Górzny ${ }^{1}$, \\ A S Walton ${ }^{1}$ and S D Evans ${ }^{1}$ \\ ${ }^{1}$ School of Physics and Astronomy, University of Leeds, West Yorkshire, UK \\ ${ }^{2}$ Department of Chemical Engineering, University of Michigan, Ann Arbor, MI 48109, USA \\ E-mail: s.d.evans@leeds.ac.uk
}

Received 1 October 2007, in final form 20 December 2007

Published 1 February 2008

Online at stacks.iop.org/Nano/19/085201

\begin{abstract}
We present an improved method for fabricating tungsten STM tips for measuring nanoscale objects using a multiprobe STM. The tips were prepared by a 'drop-off' technique combined with a magnetic field. For magnetic field strengths above 150 Oe the electrolyte was seen to rotate about the tungsten anode. This rotation reduced the adhesion of bubbles and led to a reduction in the cone angle of the tips produced. This property is advantageous for multiprobe measurements as it allows one to bring multiple tips into close proximity.
\end{abstract}

\section{Introduction}

Recent years have witnessed a dramatic increase in the production of nanostructured materials with interesting electrical and optical properties, and with them the concomitant need for characterization. Probing the electronic properties of devices on the nanoscale, however, is difficult and consequently various strategies have been devised to address this. These include:

(i) maneuvering of objects between two electrodes using atomic force microscopy (AFM) tips to create a circuit [1], (ii) lithographic patterning of electrodes onto nano-objects [2], (iii) the use of a conductive AFM tip as an electrode [3], (iv) the use of an ion beam to deposit electrodes [4], and finally (v) the use of multiprobe STMs [5].

The multiprobe STM offers several advantages over the other techniques described above. In particular it does not require a clean room for electrode fabrication and it can be performed relatively quickly in comparison with the other techniques.

The requirements for obtaining a four-probe measurement on a nano-object are as follows:

(i) Independent control of all 4 probes. (ii) In situ high resolution SEM to allow the positioning of the STM tips with high accuracy. (iii) Highly tapered tips for making contact with nanostructures and which allow multiple tips to work in close proximity.

Requirements (i) and (ii) can be met using an Omicron UHV nanoprobe system. This paper is concerned with point (iii) on how to make and improve the quality of highly tapered tips.

The most widely used method of making tips for STM is based on electrochemical etching [6-8, 14-21]. In 1987 Bryant et al [6] introduced a 'drop-off' technique in which tips were immersed into an electrolyte and etched. This technique has been adapted and modified in a variety of ways, the most significant of which has been the introduction of a high speed 'shut-off' of the etching current by Ibe et al [12]. The rapid 'shut-off' prevents any further etching of the tip after formation, implying that there will be no blunting of the tip occurring after 'drop-off'. More recently, Nishino et al [23] have adapted the 'drop-off' technique used by Bryant et al [6] to form tips made from gold to which they have attached a single fullerene to the tip; although this technique produces a very sharp tip with a small cone angle it is unlikely that such tips would be suitable for four-probe studies due to a lack of durability. Other more dated techniques for tip formation based on the grinding, cutting or breaking of tungsten wires have been reported in the literature [9-12]. However, electrochemical etching has become the preferred method. Most STM tips are fabricated from tungsten (W), platinum-iridium (Pt-Ir), or gold $(\mathrm{Au})$. These materials are selected for varying reasons. Tungsten is widely used due to its rigidity, ease of etching due to the availability of non-toxic etching solutions, and the low cost of production. Platinumiridium and gold are selected for use because they do not form oxide layers; however, cost of production can be high and uniformity of tip shape is poor [22]. 


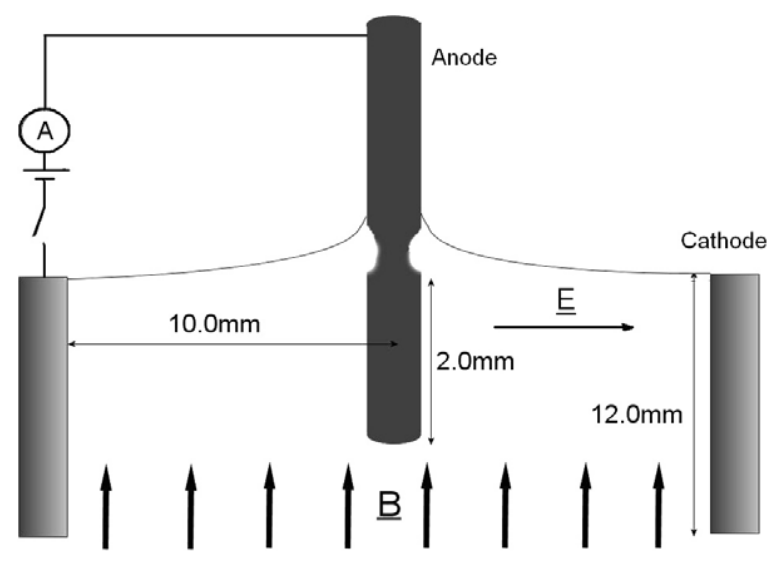

Figure 1. The experimental tip-etching configuration showing the direction of the magnetic and electric field (not to scale). The tip is inserted into the electrolyte to a depth of $2 \mathrm{~mm}$.

The ideal geometric properties for a multiprobe STM tip are that it is long and tapered (longer than $177 \mu \mathrm{m}$ for the Omicron nanoprobe) with as small a cone angle as is possible, to allow the tips to be used in close proximity. The tip should be conducting and relatively tough, to withstand physical contact with objects. Finally, having high symmetry tips is also desirable as this makes it easier to determine the position of each tip when approaching or making contact with surfaces or objects.

In this study we make use of the direct current electrochemical etching process as described by Ibe et al [12] that prevents the continued etching of a tip once 'drop-off' has occurred. Here we modify this process by introducing a magnetic field, along the tip direction. The combined electric and magnetic fields create a flow in the plane of the surface towards the cathode and removes any gas bubbles that may otherwise collect at the tip and prevent uniform tip production.

\section{Experimental details}

A $0.25 \mathrm{~mm}$ diameter temper annealed tungsten wire of $99.95 \%$ purity (supplied by Advent Research Materials) was cut to a length of 20-30 mm and sonicated in acetone for $15 \mathrm{~min}$. In the etch configuration used the tungsten wire was immersed to a depth of $2.0 \mathrm{~mm}$ in $4 \mathrm{M} \mathrm{NaOH}$. A depth of $2.0 \mathrm{~mm}$ was found to be optimal for a clean 'drop-off' to occur. The wire was connected as an anode and located centrally within a cylindrical cathode (stainless steel) of diameter $20 \mathrm{~mm}$. Figure 1 shows schematically the arrangement used for tip fabrication. The $\mathrm{NaOH}$ depth was adjusted to be level with the cathode.

The potential between the anode and the cathode was supplied using a home-built apparatus similar in design to that described by Ibe et al [12].

The electrochemical circuit used a high speed comparator to drive two complementary transistors. These, combined with a small resistor, reduced the current in the 'cut-off' phase with a 500 ns 'cut-off' time once the current had dropped below a set point (typically $4.0 \mathrm{~mA}$ ). This rapid termination of the current prevented any continuation of the etching process.

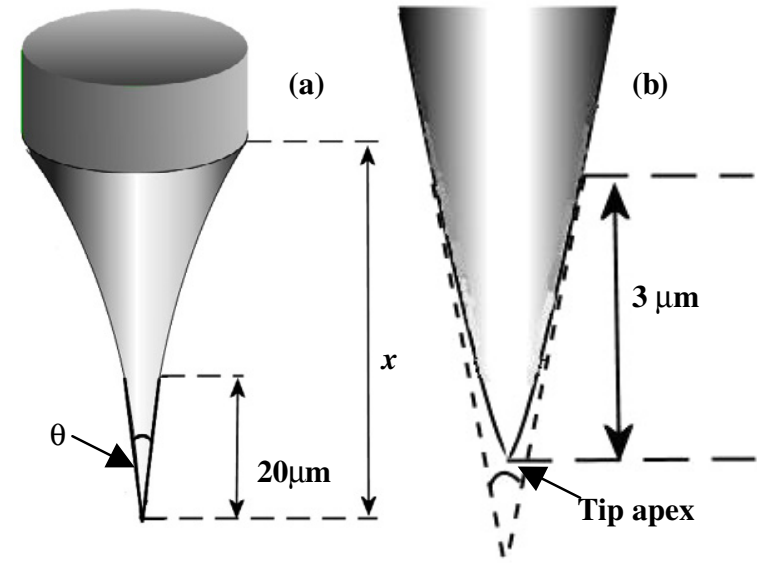

Figure 2. (a) The method used for defining tip parameters at low magnifications. The tip length is defined as the distance between the highest point of etch and the tip apex and is represented by $x$. The cone angle $\theta$ is defined as that subtended between tangents on each side of the tip (at a height of $20 \mu \mathrm{m}$ from the apex) originating at the apex. (b) The method used for defining the cone angle at high magnifications. The cone angle is taken as an extrapolation of tangents from the cone at $3 \mu \mathrm{m}$ due to its hyperbolic shape.

A magnetic field was applied parallel to the wire (anode) (and perpendicular to the air-electrolyte interface), using an electromagnet (RS $12 \mathrm{~V}$ holding magnet). The magnitude of the field strength at the tip position could be varied between 0 and 230 Oe by changing the voltage between 0 and $15 \mathrm{~V}$.

After formation the tips were rinsed with deionized water to remove any remaining $\mathrm{NaOH}$ solution and imaged using a Zeiss Gemini 1500 SEM.

\section{Results and discussion}

The length and cone angle of the tips were investigated as a function of magnetic field. The length and cone angle are defined in figure 2.

Experimentally SEM was used to determine the tip lengths (from the $600 \times$ magnification) and cone angles (on both the $3000 \times$ and $30000 \times$ magnifications). Typical images of a good tip are shown in figures 3(a)-(c) at the three magnifications used for data analysis. The cone angles were measured using images of equal magnification to figures 3(b) and (c).

The magnitude of the magnetic field was varied between 0 and 230 Oe whilst the tip immersion depth was maintained at $2.0 \mathrm{~mm}$ and the 'cut-off' current at $4 \mathrm{~mA}$. The magnetic field caused the electrolyte to rotate, in an anticlockwise direction, for field strengths greater than $125 \mathrm{Oe}$. The electrolyte was imaged using a NAC 500 high speed digital camera and bubbles evolving from the anode were observed to rotate at $(14.1 \pm 0.9) \mathrm{mm} \mathrm{s}^{-1}$ for a magnetic field strength of 230 Oe. The rotational speed of the electrolyte was seen to increase linearly with magnetic field for field strengths greater than 125 Oe.

An initial investigation was implemented in which measurements were taken on a sample of 80 tips ( 40 produced with a 180 Oe magnetic field and 40 with no field). A further 

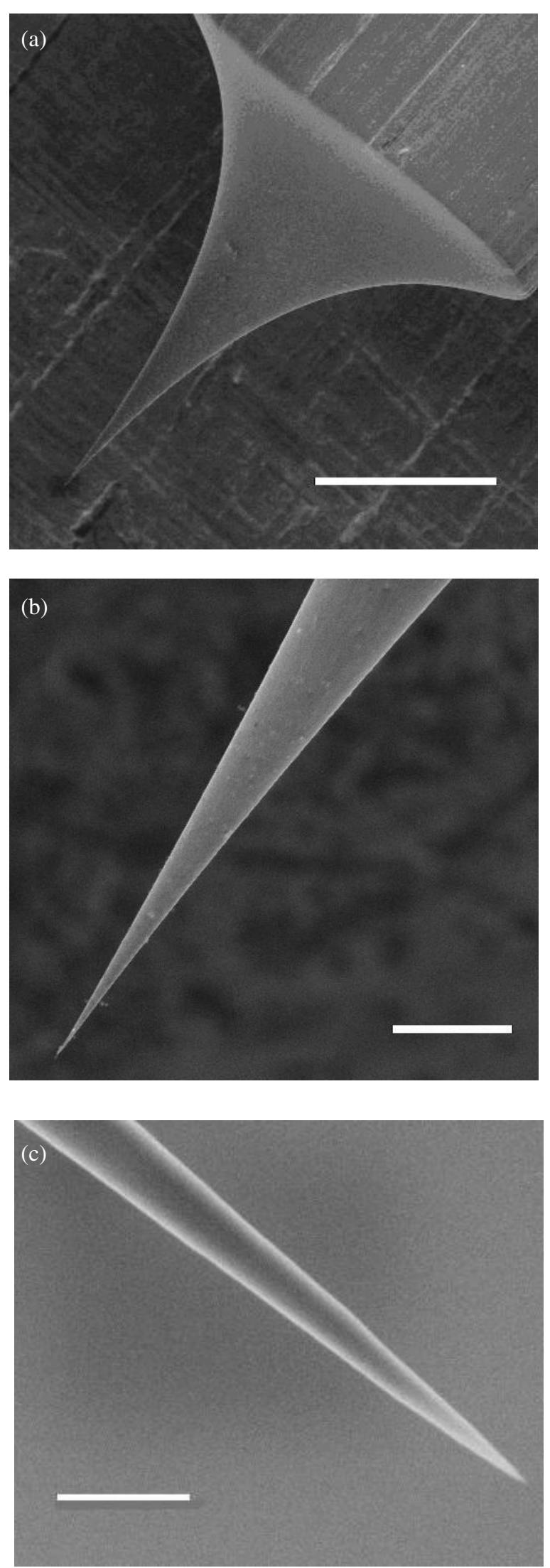

Figure 3. (a) A SEM image of a tungsten tip taken at $600 \times$ magnification; the white bar represents a length of $100 \mu \mathrm{m}$. (b) A SEM image of a tungsten tip at $3000 \times$ magnification. The white bar represents a length of $10 \mu \mathrm{m}$. (c) A SEM image of a tungsten tip at $30000 \times$ magnification. The white bar represents a length of $1 \mu \mathrm{m}$.

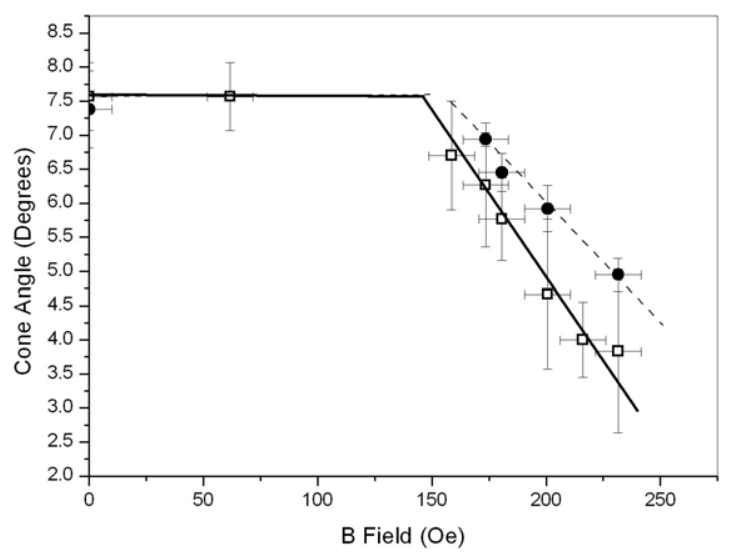

Figure 4. Graph showing the variation in cone angle as a function of the magnetic field. The solid circle points represent cone angles measured at $30000 \times$ magnification. The hollow square points represent cone angles measured at $3000 \times$ magnification. The lines are guides to the eye.

investigation into the effect of the magnitude of the magnetic field on tip geometry was also performed (figure 4). Tips produced with a magnetic field showed a modest increase in length (from $262 \pm 44 \mu \mathrm{m}$ to $298 \pm 31 \mu \mathrm{m}$ ). More importantly, however, the cone angle showed a significant decrease from $7.4^{\circ} \pm 0.5^{\circ}$ to $3.9^{\circ} \pm 1.3^{\circ}$. The radii of the tips produced with a magnetic field of 180 Oe were found to be $21.3 \pm 5.4 \mathrm{~nm}$, measured at $45000 \times$ magnification, this represents a small but systematic reduction in comparison with tips made without a magnetic field $(28.2 \pm 14.7 \mathrm{~nm})$. In addition to the decrease in cone angle it was found that the tips produced in the presence of a magnetic field showed greater axial symmetry and an improved rate of 'good' tip production.

Typically a potential of $6 \mathrm{~V}$ is applied between the tungsten wire (anode) and the stainless steel (cathode). The cylindrical symmetry ensures that the electric field, within the electrolyte, is directed radially towards the cathode. In $\mathrm{NaOH}$ solution, reactions at the anode and cathode lead to the production of water-soluble sodium tungstate and hydrogen [12]:

$$
\begin{aligned}
& \text { cathode: } 6 \mathrm{H}_{2} \mathrm{O}+6 \mathrm{e}^{-} \rightarrow 3 \mathrm{H}_{2}(\mathrm{~g})+6 \mathrm{OH}^{-} \\
& \text {anode: } \mathrm{W}(\mathrm{s})+8 \mathrm{OH}^{-} \rightarrow \mathrm{WO}_{4}^{2-}+4 \mathrm{H}_{2} \mathrm{O}+6 \mathrm{e}^{-} \\
& \mathrm{W}(\mathrm{s})+2 \mathrm{OH}^{-}+2 \mathrm{H}_{2} \mathrm{O} \rightarrow \mathrm{WO}_{4}^{2-}+3 \mathrm{H}_{2}(\mathrm{~g}) .
\end{aligned}
$$

Hydrogen gas evolves at the cathode rather than the anode [13]. However, owing to the high potential at which the tips are etched it is likely there will be disassociation of the water, leading to oxygen bubbles being formed at the anode (tungsten wire). These bubbles often adhere to the tungsten wire thereby interfering with the etching process (and leading to irregular tip formation). In the presence of a magnetic field (directed normal to the air/solution interface) we find that the bubbles move with the electrolyte and spiral out towards the cathode under the influence of the Lorentz force $(\mathbf{F}=q \mathbf{E}+$ $\left.\mathbf{v}^{\curlywedge} \mathbf{B}\right)$ acting upon the electrolyte. For magnetic fields $>150 \mathrm{Oe}$ we find that the Lorentz force is sufficient to effectively prevent the adhesion of macroscopic bubbles, rapidly sweeping them to 

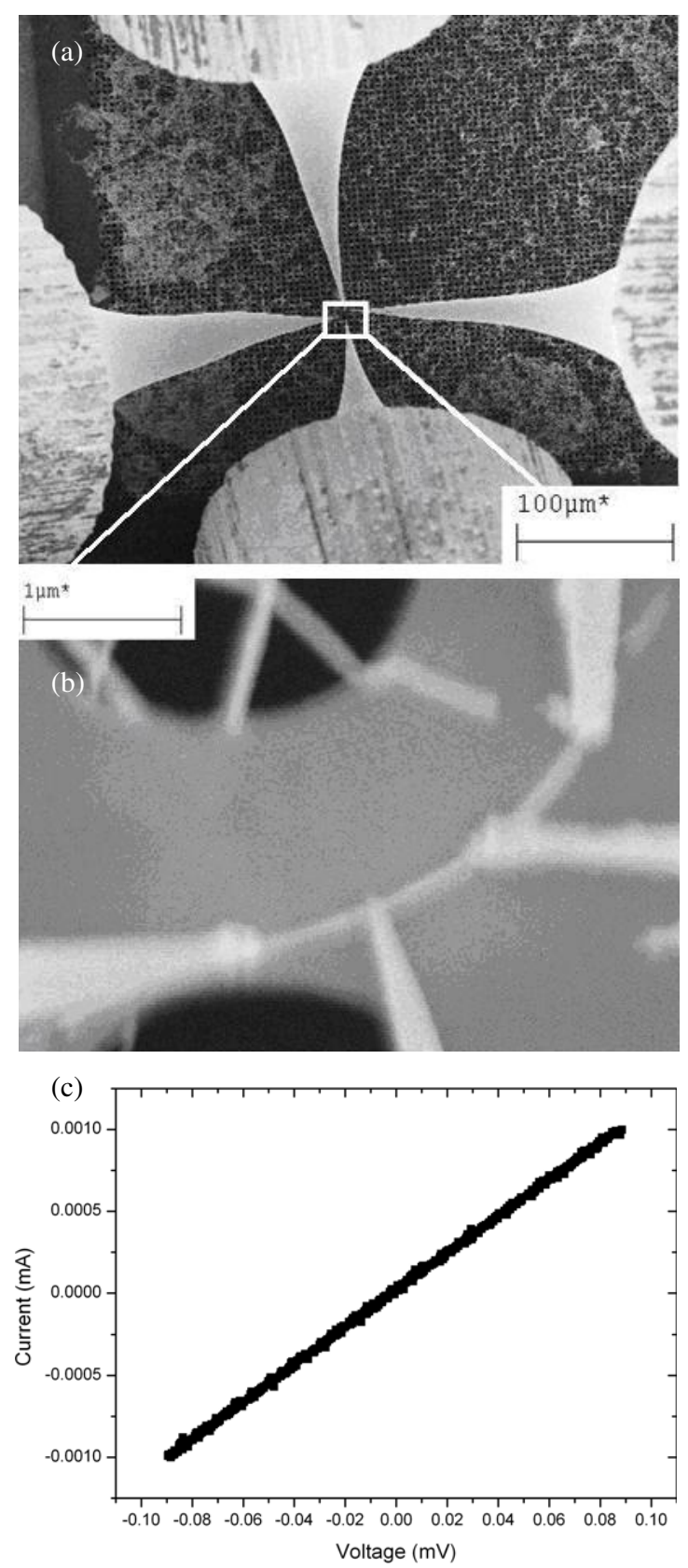

Figure 5. (a) Four probes brought into contact with $50 \mathrm{~nm}$ diameter gold wire. (b) High resolution image of four probes in contact with $50 \mathrm{~nm}$ diameter gold wire. (c) $I-V$ sweep giving a resistance of $940 \Omega$.

the cathode thus producing an alteration in tip shape (to a more parabolic profile with a reduced cone angle) increasing the symmetry of the tips and increasing successful tip production to greater than $90 \%$. The tips produced in this way have been shown to be suitable for electrical characterization on nanowires.

Figure 4 shows the variation of cone angle and magnetic field. The initial plateau shows the magnetic field to have little or no effect at field strengths of less than 150 Oe. Above 150 Oe the cone angle decreases linearly. Optical visualization of the electrolyte during tip formation showed it to rotate about the wire. For fields greater than 150 Oe the rate of rotation of the fluid was $540 \mathrm{rpm}$ or greater. This created sufficient force to prevent the small bubbles formed during etching from adhering to the tip surface.

In order to verify that the tips produced in this way are appropriate for the electrical probing of nanostructures we show in figure 5 an $I-V$ measurement on a $50 \mathrm{~nm}$ diameter gold nanowire. The technique used to obtain the measurement is very similar to that described by Walton et al [5], with the exception that here the nanowires were deposited on a silicon nitride TEM grid with $2 \mu \mathrm{m}$ holes. The grids allow the probes to be brought into contact with the nanowires with relative ease, since they make it easier to determine the height of the tips relative to the substrate.

Using carbon nanotube tips Murata et al [24] have reported a minimal achievable tip separation of $50 \mathrm{~nm}$, limited in their case by the resolution of their SEM. The Zeiss Gemini 1500 used in our system has a resolution of less than $3 \mathrm{~nm}$, which in conjunction with the tips typically used here (cone angle $3.9^{\circ}$, tip radius $21.3 \mathrm{~nm}$ ) allows contact down to $9.1 \mathrm{~nm}$. This is a factor of two improvement upon that reported by Nagase et al [25].

A typical $I-V$ sweep, as in figure 5(c), shows the gold nanowires to have ohmic properties. The resistivity of the wires has been determined as $(2.1 \pm 0.4) \times 10^{-7} \Omega \mathrm{m}$, whereas the bulk resistivity of gold is $2.05 \times 10^{-8} \Omega \mathrm{m}$.

The larger than expected value for the resistivity of the wires is likely to be due to an increase in the surface scattering. A high polycrystallinity, causing electron scattering at grain boundaries and impurities in the wire, could also contribute to the observed increase in resistivity. Walton et al [5] also found a larger than expected resistivity $(2.5 \pm 0.1) \times 10^{-8} \Omega \mathrm{m}$; however, the wires measured here have a considerably smaller diameter.

The results from the four-probe measurement show that the tips produced using these techniques are highly suitable for multiprobe STM studies of electronic devices on the nanoscale.

\section{Conclusions}

In this work we have investigated the effect of varying the magnitude of a magnetic field on tip production. For fields greater than 150 Oe the electrolyte was found to rotate about the anode. This rotation resulted in the removal of bubbles from the etching tungsten wire and was found to significantly improve the tips produced. The resulting tips had a reduction in cone angle of up to $3.5^{\circ}$, a higher degree of symmetry and an increased rate of successful production. While the study has focused on the anodic dissolution of tungsten wire with $\mathrm{NaOH}$, we believe that these results will be generally applicable to other metal etchant systems.

\section{Acknowledgments}

We acknowledge the University of Leeds, the EPSRC and the Rothschild Community of Excellence for their support and funding. 


\section{References}

[1] Avouris Ph, Hertel T, Martel R, Schmidt T, Shea H R and Walkup R E 1999 Appl. Surf. Sci. 141201

[2] Tang J, De Poortere E P, Klare J E, Nuckolls C and Wind S J 2006 Microelectron. Eng. 831706

[3] Ishida T, Mizutani W, Liang T T, Azehara H, Miyake K, Sasaki S and Tokumoto H 2003 Ann. New York Acad. Sci. $1006164-86$

[4] Hayes A V 2000 Rev. Sci. Instrum. 711163

[5] Walton A S, Allen C S, Critchley K, G'orzny M Ł, McKendry J E, Brydson R M D, Hickey B J and Evans S D 2007 Nanotechnology 18065204

[6] Bryant P J, Kim H S, Zheng Y C and Yang R 1987 Rev. Sci. Instrum. $\mathbf{5 8} 1115$

[7] Heben M J, Penner R M, Lewis N S, Dovek M M and Quate C F 1988 J. Microsc. 152651

[8] Nagahara L A, Thundat T and Lindsay S M 1989 Rev. Sci. Instrum. 603128

[9] Binnig G, Rohrer H, Gerber C and Weibel E 1982 Phys. Rev. Lett. 4957

[10] Binh V T 1988 J. Microsc. 152355

[11] Stupian G W and Leung M S 1989 Rev. Sci. Instrum. 60181
[12] Ibe J P, Bey P P Jr, Brandow S L, Brizzolara R A, Burnham N A, DiLella D P, Lee K P, Marrian C R K and Colton R J 1990 J. Vac. Sci. Technol. A 83570

[13] Fotino M 1993 Rev. Sci. Instrum. 64159

[14] Kar A K, Gangopadhyay S and Mathur B K 2000 Meas. Sci. Technol. 111426

[15] Huang L and Lee Y H 1993 Rev. Sci. Instrum. 643022

[16] Klein M and Schwitzgebel G 1997 Rev. Sci. Instrum. 683099

[17] Muller A D, Muller F and Hietschold M 1999 Rev. Sci. Instrum. 703970

[18] Fotino M 1993 Rev. Sci. Instrum. 64159

[19] Quaade U J and Oddershede L 2002 Europhys. Lett. 57611

[20] Ottaviano L, Lozzi L and Santucci S 2003 Rev. Sci. Instrum. 743360

[21] Olivia A I, Romero A and Pena J L 1996 Rev. Sci. Instrum. 671917

[22] Ren B, Picardi G and Pettinger B 2004 Rev. Sci. Instrum. 75837

[23] Nishino T, Ito T and Umezawa Y 2005 Proc. Natl Acad. Sci. 1025661

[24] Murata Y et al 2005 Japan. J. Appl. Phys. 445336

[25] Nagase M and Yamaguchi H 2007 J. Phys. Conf. Ser. 61856 